\title{
STK31 as novel biomarker of metastatic potential and tumorigenicity of colorectal cancer
}

\author{
Lan Zhong ${ }^{1, *}$, Jing Liu ${ }^{2, *}$, Yedong Hu${ }^{1}$, Wei Wang ${ }^{1}$, Fei $X_{u^{1}}$, Wen $X u^{1}$, Junyi Han ${ }^{3}$, \\ Ewelina Biskup ${ }^{4}$ \\ ${ }^{1}$ Department of Gastroenterology, East Hospital of Tongji University School of Medicine, Shanghai, China \\ ${ }^{2}$ State Key Laboratory of Genetic Engineering, Institute of Genetics, School of Life Sciences, Fudan University, Shanghai, \\ China \\ ${ }^{3}$ Department of General Surgery, East Hospital of Tongji University School of Medicine, Shanghai, China \\ ${ }^{4}$ Department of Internal Medicine, University Hospital of Basel, Switzerland \\ *These authors contributed equally to this work \\ Correspondence to: Junyi Han, email: doctortonyhjy@163.com
}

Keywords: colorectal cancer, metastasis, serine-threonine kinase 31 (STK31), cancer testis antigen

Received: August 25, $2016 \quad$ Accepted: February 01, $2017 \quad$ Published: February 16, 2017

Copyright: Zhong et al. This is an open-access article distributed under the terms of the Creative Commons Attribution License (CC-BY), which permits unrestricted use, distribution, and reproduction in any medium, provided the original author and source are credited.

\section{ABSTRACT}

Purpose: Colorectal cancer (CRC) is the fifth most common cause of cancer deaths in China and fourth worldwide. Metastatic dissemination of primary tumors is considered main cause for CRC related mortality. The serine-threonine kinase 31 (STK31) gene is a novel cancer testis (CT) antigen. It was found significantly highly expressed in gastrointestinal cancers. In our study we aimed to analyze the correlation between STK31 expression patterns and metastasization, tumor stage and grade in CRC patients.

Results: Relative STK31 expression level was significantly higher in patients with lymph node metastasis. STK31 expression levels in primary tumorous tissues of metastatic patients were significantly higher than in ANCTs and in lymph nodes samples, both at the RNA level and the protein level.

Materials and Methods: Surgical specimens of cancerous tissues, paired with adjacent noncancerous tissues, and lymph nodes from 44 CRC cases with different clinicopathological features were collected. Expression of STK31 was detected and measured by immunohistochemistry and quantitative real-time polymerase chain reaction (QRT-PCR).

Conclusions: Our data suggest that STK31 might be a potential biomarker in detecting, monitoring and predicting the metastatic risk of colorectal cancer.

\section{INTRODUCTION}

Modern diet and lifestyle contributed greatly towards a raise of colorectal cancer (CRC) occurrence, which became an emergent health care burden worldwide. Its global incidence continues to increase, especially in developed, welfare countries. GLOBOCAN 2012 (International Agency for Research on Cancer, http:// globocan.iarc.fr/Default.aspx) estimated over 373631 CRC deaths, accounting for $8 \%$ of all cancer deaths, positioning $\mathrm{CRC}$ as the fifth most common cause of cancer deaths in China and the fourth worldwide. The golden clinical standard for colorectal cancer prophylaxis and diagnostics is colonoscopy. Because of its invasive nature and insidious, often silent progression, the majority of CRC patients are diagnosed only at an advanced stages [1], where therapeutic regimens are limited and prognosis poorly. If detected early enough, the tumor can be treated with excellent results, providing the patient with a good long-term prognosis and long recurrence-free interval. Thus, systematic primary prophylaxis, early diagnosis and rapid intervention are critical for reducing CRC mortality $[2,3]$.

Despite a growing number of genes identified and investigated as biomarkers in CRC, such as SPAG9 or PTCH1 $[1,4]$, none is routinely used for clinical detection and anticancer treatment. Therefore, it is vital to explore 
biomarkers more profoundly and design simple methods to implement them into prophylactic the therapeutic, as well as monitoring and prognostic approaches. Especially useful would be the application of genetic biomarkers to detect and predict $\mathrm{CRC}$ metastasis. Identification of specific measurable molecules that are associated with tumor aggressiveness would be of great clinical significance, providing options for assessing early spread of CRC.

The serine-threonine kinase 31 (STK31) gene was initially identified through cDNA subtraction as a testis-specific protein kinase gene expressed in mouse spermatogonia [5, 6]. Immunolocalization of equine STK31 revealed a specific localization in the equatorial region of post-meiotic spermatocytes, elongating spermatids, and ejaculated spermatozoa [7]. Human STK31 gene, mapped to chromosome $7 \mathrm{p} 15.3$, has two splice variants reported to date, which encode a putative 1,019 a.a. protein (isoform a; accession \#NP113602) and a putative 996 a.a. protein (isoform b; accession \#NP116562). Although the biological function of STK31 remains to be determined, its highly conserved domain structure between vertebrate species, including a tudor domain, a SbcC domain as well as the putative serine-threonine kinase domain [7], indicates that it might be involved in DNA repairing/repackaging and chromatin remodeling during spermiogenesis, sperm maturation, and sperm function [8-11]. Recently, STK31 has been described as a novel cancer testis (CT) antigen, highly expressed in GI cancer cells (colorectal, gastric and esophageal cancer), while restricted to testis and fetal brain in normal tissues [12]. Mori et al [12] reported that STK31derived peptide was able to trigger specific cytotoxic T-lymphocytes (CTLs) and to induce CTLs cell lysis in a HLA-A*0201-restricted manner or by peptide-loading. STK31 is thus a potential diagnostic biomarker for CRC, a good candidate for targeted therapy and monitoring, as well as an assumptive predictive and prognostic factor for early stage and metastatic CRC.

In the present study, we aimed to conduct an accurate quantification of STK31 in order to investigate the correlation of its expression and CRC features. Besides of immunohistochemistry, we also carried out a realtime RT-PCR to measure mRNA expression in human CRC tissues and their adjacent non-cancerous samples. Our findings demonstrated a close relationship between STK31 expression between tumor stage and lymph node metastasis in colorectal cancer, suggesting a potential functional role in tumor progression.

\section{RESULTS}

\section{Relative quantification of STK31 mRNA expression in tumors and adjacent noncancerous tissues}

mRNA levels of STK31 were measured by quantitative real-time RT-PCR. Measurements were normalized using cycle threshold (CT) method. STK31 mRNA in the target tissue was related to the paired internal control and calculated as $2^{-\Delta \mathrm{CT}}(\Delta \mathrm{CT}=\mathrm{CT}(\mathrm{STK} 31)-\mathrm{CT}$ (GAPDH)). When compared, STK31 expression level in $\mathrm{CRC}$ tissues and that in adjacent noncancerous tissues (ANCTs) differed significantly.

\section{STK31 mRNA expression in relation to lymph node status}

We divided the patients into two groups according to their lymph node (LN) metastatic status. Patients without metastatic lymph nodes were placed in group A and patients with metastatic lymph nodes in group B. We found a significantly higher relative STK31 expression in primary tumor samples from patients with metastasis (group B) than in those without metastasis (group A) $((0.00778 \pm 0.0108$ VS $0.00240 \pm 0.0531, P=0.003$, Figure 1), confirmed by stratified expression level analysis.

In group A (non-LN-metastatic patients), there was no statistically significant correlation between tumor tissues and ANCTs $(p=0.255)$. In group B (metastatic group), STK31 expression levels in the primary tumorous tissues were much higher than in ANCTs and lymph nodes (Figure 2). Statistical One-way ANOVA analysis performed on group B (metastatic patients) revealed a significant difference between tumors, ANCTs and lymph nodes (ANOVA between groups $\mathrm{F}=0.021$; between tumors and ANCTs $p=0.009$, between tumors and lymph nodes $p=0.031$ ).

The relationship between STK31 mRNA expression and clinico-pathological features in group B patients is summarized in Table 1. Significant correlations were seen for male sex, age of less than 60 years, well or moderately differentiated histologic grade, and serosal invasion (T3 stage). These data suggest a close relationship between STK31 expression and tumor cell migration and invasion (metastatic potential).

\section{Relationship between STK31 mRNA expression level and further clinicopathological features}

Statistical analysis of STK31 expression in groups of patients divided according to their clinicpathological parameters (and not to the LN metastatic status) showed no correlation between the STK31 mRNA levels and features, such as age, gender, tumor site, histologic grade, primary tumor invasion depth, or Duke's stage of the carcinoma (data not shown).

\section{Immunobiological measurement of STK31 protein expression in tumor patients with lymph node metastasis}

STK31 expression was observed in membrane of tumor cells after immunohistological staining (Figure 3A). 
The relative expression of STK31mRNA in PT with/without metastasis

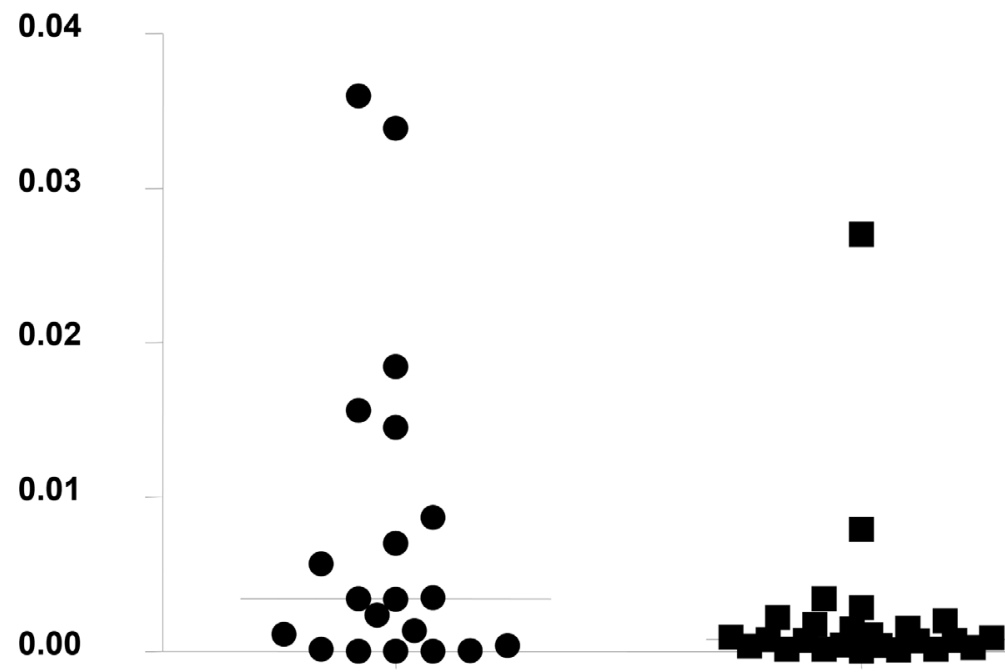

PT with metastasis

PT without metastasis

Figure 1: The relative STK31 mRNA expression in primary tumor samples from patients with metastasis is significantly higher than in those without metastasis $(0.00778 \pm 0.0108$ VS $0.00240 \pm 0.0531, P=0.003)$.

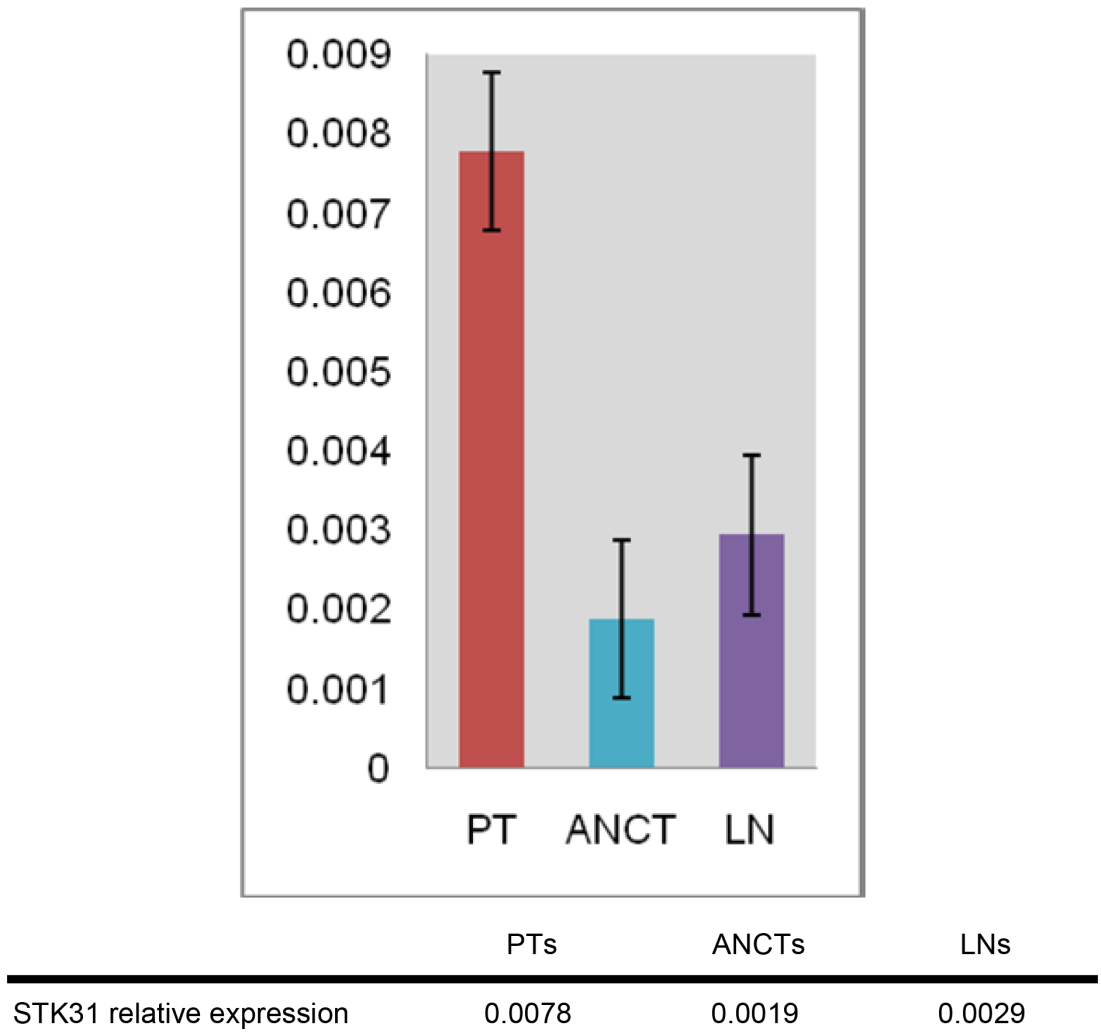

Figure 2: STK31 mRNA levels in tumor samples, adjacent noncancerous tissues and lymph nodes in CRC patients with metastasis, as determined by real-time RT-PCR. PT: primary tumorous tissue; ANCT: adjacent noncancerous tissue; LN: lymph node. 
Table 1: Association between STK31 expression and clinicopathologic variables in metastatic CRC patients using One-way ANOVA analysis

\begin{tabular}{|c|c|c|c|c|c|}
\hline \multirow[t]{2}{*}{ clinicopathologic features } & \multirow[t]{2}{*}{$n$} & \multirow{2}{*}{$\begin{array}{c}F \text { value } \\
\text { ( } P \text { value between groups) }\end{array}$} & \multicolumn{3}{|c|}{$P$ value within groups } \\
\hline & & & PT vs. ANCTs & PT vs. LNs & ANCT vs. LNs \\
\hline \multicolumn{6}{|l|}{ Total } \\
\hline & 20 & $0.021^{*}$ & $0.009 *$ & $0.031 *$ & 0.630 \\
\hline \multicolumn{6}{|l|}{ Gender } \\
\hline Male & 14 & $0.033^{*}$ & $0.024 *$ & $0.023^{*}$ & 0.991 \\
\hline Female & 6 & 0.415 & 0.192 & 0.508 & 0.502 \\
\hline \multicolumn{6}{|l|}{ Age } \\
\hline$\leq 60$ & 14 & $0.043 *$ & $0.027 *$ & $0.032 *$ & 0.935 \\
\hline$>60$ & 6 & 0.371 & 0.168 & 0.414 & 0.551 \\
\hline \multicolumn{6}{|l|}{ Tumor site } \\
\hline Colon & 8 & 0.099 & 0.076 & 0.052 & 0.853 \\
\hline Rectum & 12 & 0.181 & 0.068 & 0.268 & 0.453 \\
\hline \multicolumn{6}{|l|}{ Histologic grade } \\
\hline Well/moderately differentiated & 15 & $0.025^{*}$ & $0.017 *$ & $0.019^{*}$ & 0.957 \\
\hline Poorly differentiated & 5 & 0.520 & 0.263 & 0.585 & 0.551 \\
\hline \multicolumn{6}{|l|}{ T stage } \\
\hline $\mathrm{T} 2$ & 3 & 0.428 & 0.211 & 0.495 & 0.526 \\
\hline T3 & 12 & $0.015^{*}$ & $0.015^{*}$ & $0.009^{*}$ & 0.824 \\
\hline $\mathrm{T} 4$ & 5 & 0.650 & 0.975 & 0.421 & 0.438 \\
\hline \multicolumn{6}{|l|}{ Tumor stage } \\
\hline Stage I + II & 12 & 0.093 & $0.033^{*}$ & 0.158 & 0.439 \\
\hline Stage III + IV & 8 & 0.069 & 0.102 & $0.026^{*}$ & 0.498 \\
\hline
\end{tabular}

Three groups: PT: primary tumorous tissue; ANCTs: adjacent noncancerous tissues; LNs: lymph nodes. ${ }^{*} P<0.05$ (statistically significant).

Consistently with the mRNA expression patterns, STK31 protein expression levels in tumor tissues of non-metastatic patients were much lower (Figure 3B). Furthermore, our immunohistochemistry staining also confirmed that the STK31 is expressed higher in primary tumorous tissues of patients with metastasis (Figure 3C) were significantly higher than in paired adjacent noncancerous tissues Figure 3D) and in lymph nodes Figure 3E).

\section{DISCUSSION}

CRC is a malignancy with one of the highest incidences and mortalities, especially in developed countries, and represents a severe public health issue worldwide. In the past decades, CRC morbidity and mortality increased rapidly in China [4]. Despite a great improvement in diagnostics and treatment (novel chemotherapeutics, immunotherapy and molecular targeted therapy), the majority of CRC patients are diagnosed only at advanced stages and the 5-year mortality stagnates at approximately $40 \%$ [13-16]. Cell migration and invasion is a critical event in carcinogenesis and cancer progression, resulting in distant metastasis, especially to the liver $[1,17]$. It has been reported that approximately $90 \%$ of CRC deaths arise from the metastatic dissemination of primary tumors, and distant metastasis has been regarded as the main cause of death in CRC patients [17-19]; still remains a challenge for oncologists to predict local recurrence, metastatic potential and outcome of primary colorectal carcinoma patients based on conventional clinical and histopathological/immunhistochemical exams $[2,20]$. To date, tumor-node-metastasis (TNM) classification represents the main tool for identifying prognostic differences among patients with early-stage CRC [21]. Numerous approaches have been undertaken to characterize tumor-associated antigens. However, only few proteins have been proven to be useful biomarkers (carcinoembryonic antigen CEA, CA19.9 and CA125) [22] ). While they are applied in therapy monitoring and recurrence diagnosis, their validity in clinical screening, 
cancer grading and metastatic potential evaluation is not sufficient. Therefore, in order to improve treatment efficiency and efficacy, identification of new reliable, applicable and cost effective markers to detect, predict and monitor CRC metastasis in clinical practice.

We examined the clinical relevance of STK31 as a potential predictor of CRC cell migration. Our data revealed that, as a member of the CT gene family that encodes unique class of tumor-associated antigens in various malignancies, STK31 is closely related with CRC metastasis. STK31 expression in primary tumor samples of patients with lymph node metastasis was significantly higher than in those without metastasis. Moreover, STK31 expression levels in primary cancer tissues of metastatic patients were significantly higher than in ANCTs and in lymph nodes, both at mRNA and protein level. These results not only indicated that STK31 plays an important role in cell migration and invasion, but also suggested that STK31 could be a potential tumor biomarker for detecting, monitoring and predicting CRC metastatic risk, as well as individually tailor therapeutic strategies. Data of Mori et al. showed that over-expression of the STK31 gene did not contribute to progression of colorectal cancer [12]. However, this study compared STK31 expression levels in patients with and without metastasis using $\chi^{2}$ test based on an assumptive threshold to assign the data into different groups. We used Independent-Samples $t$ test. Additionally, there were ethnic differences between our samples. Moreover, it has been shown that STK31 knockdown lead to a significant suppression of tumorigenicity in colon cancer cells, while STK31 over-expression promotes an undifferentiated CRC status [23]. Kuo et al [24]. proved STK31 to be a cell cycle regulated centrosome associated protein. Ectopically-expressed, STK31 increases cell migration and invasiveness of human somatic cancer cells, whereas endogenous STK31 knockdown results in microtubule assembly defects that prolong the duration of mitosis and triggers apoptosis.

Furthermore, targeting STK31 mitotic kinase can be a novel anticancer treatment [25]. STK31-derived peptide elicits specific CTLs, among others in a HLA-A*0201restricted manner. Since HLA-A*0201 is the most commonly expressed allele and expression of CT antigens are normally restricted to immunologically privileged male germ cells, targeting STK31 in CRC patients may be a promising immunotherapeutic approach, especially in metastatic patients.

Our study provides firm evidence that STK31 is associated with metastatic $\mathrm{CRC}$, and that the association can be easily measured in Real-time PCR or immunohistostaining. High STK31 expression in resected tissues could assist the leading oncologist to suggest an appropriate management for individual patients. It is striking that the expression of STK31 in primary tumor is higher than in the metastatic lymph nodes. According to the study of Fok et al [23], STK31 is robustly and heterogeneously expressed in colon cancer tissues and plays a critical role in determining the differentiation

A

B

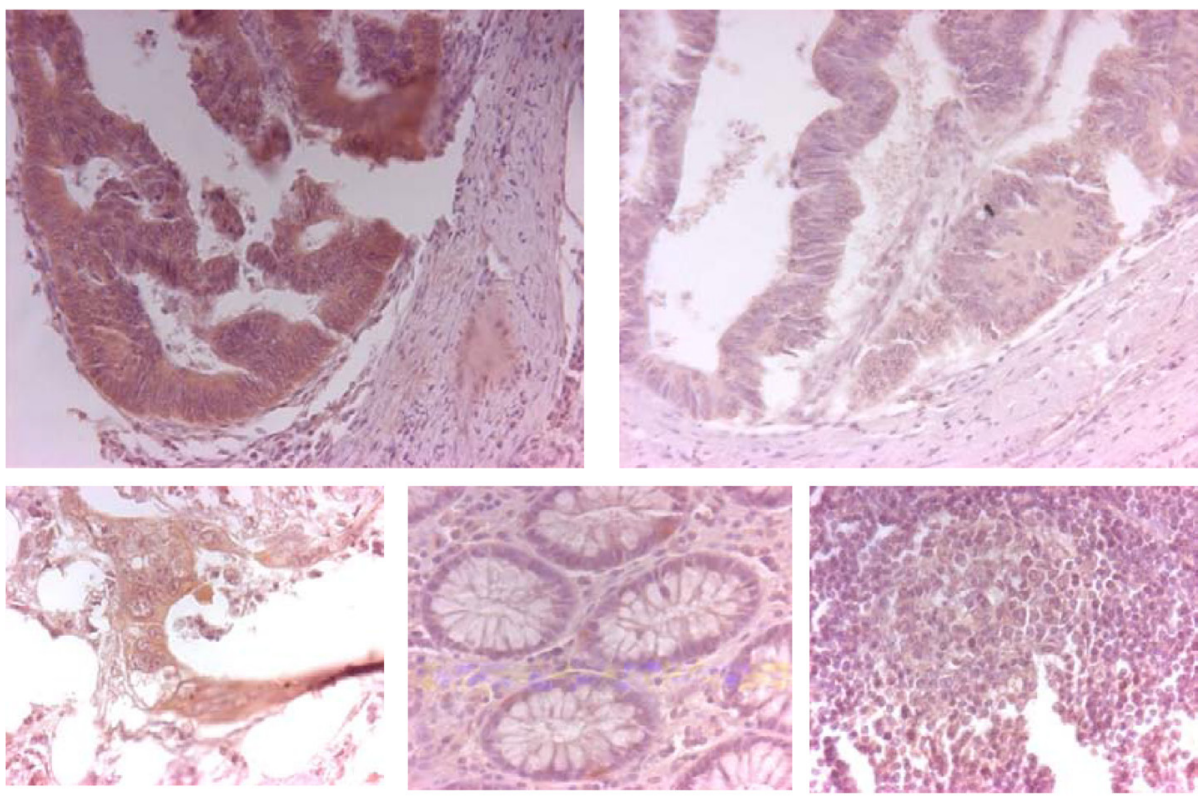

$\mathrm{C}$

$\mathrm{D}$

E

Figure 3: Immunohistochemistry. STK31 protein expression in a primary tumor tissue of a patient with lymph node metastases (A) and in a patient without metastases (B). Primary tumor samples from a patient with lymph node metastasis: (C) primary tumorous tissue (strong expression), (D) adjacent noncancerous tissue (no expression) and (E) lymph node metastasis (weak expression). 
Table 2: Clinicopathological characteristics of the study patients

\begin{tabular}{|c|c|c|c|}
\hline Characteristics & Specifics & $\begin{array}{c}\text { No. of metastatic CRC } \\
\text { patients (20) }\end{array}$ & $\begin{array}{c}\text { No. of non-metastatic CRC } \\
\text { patients (24) }\end{array}$ \\
\hline \multirow[t]{2}{*}{ Age distribution } & $\leq 60 \mathrm{y}$ & $14(70.0 \%)$ & $9(37.5 \%)$ \\
\hline & $>60 y$ & $6(30.0 \%)$ & $15(62.5 \%)$ \\
\hline \multirow[t]{2}{*}{ Gender } & Male & $14(70.0 \%)$ & $13(54.2 \%)$ \\
\hline & Female & $6(30.0 \%)$ & $11(45.8 \%)$ \\
\hline \multirow[t]{2}{*}{ CRC location } & Colon & $8(40.0 \%)$ & $8(33.3 \%)$ \\
\hline & Rectum & $12(60.0 \%)$ & $16(66.7 \%)$ \\
\hline \multirow[t]{2}{*}{$\begin{array}{l}\text { Histologic } \\
\text { grades }\end{array}$} & $\begin{array}{c}\text { Well or moderately } \\
\text { differentiated }\end{array}$ & $15(75.0 \%)$ & $20(83.3 \%)$ \\
\hline & Poorly differentiated & $5(25.0 \%)$ & $4(16.7 \%)$ \\
\hline \multirow[t]{3}{*}{ T stage } & $\mathrm{T} 2$ & $3(15.0 \%)$ & $2(8.3 \%)$ \\
\hline & $\mathrm{T} 3$ & $12(60 \%)$ & $17(70.8 \%)$ \\
\hline & $\mathrm{T} 3, \mathrm{~T} 4$ & $5(25.0 \%)$ & $5(20.8 \%)$ \\
\hline \multirow[t]{2}{*}{ Stage at diagnosis } & Stage I + II & $12(60.0 \%)$ & $13(54.2 \%)$ \\
\hline & Stage III + IV & $8(40.0 \%)$ & $11(45.8 \%)$ \\
\hline
\end{tabular}

state of colon cancer cells. Thus, the inconsistency may be related to different tumor differentiation in the primary tumor site and the lymph node metastases, Moreover, the discrepancy might underline the important role of STK1 in the early metastatic processes. Further studies on larger samples to investigate the sensitivity and specificity of STK31 for predicting metastatic CRC risk are needed. Measurements of mRNA and STK31 protein levels in tumor cells with high and low metastatic potential by RTPCR, immunohistochemistry analysis and flow cytometry are warranted. Moreover, exploration of the prognostic value of STK31 expression for metastasis formation and predictive value for overall- and disease-free survival (DFS) after a long-term follow-up is ongoing.

In conclusion, our study is to our knowledge the first attempt to analyze STK31 expression in Chinese CRC population in relation to different clinicopathological features. We found a higher relative STK31 expression level in patients with lymph node metastasis, and demonstrated the diagnostic and prognostic potential of STK31 transcripts in colorectal cancer patients. High STK31 levels in the primary tumor may be prognostic for high risk of metastasis and correlate with an increased patient mortality, rendering it a potential prognostic factor, biological marker of tumor invasiveness and therapeutic target.

\section{MATERIALS AND METHODS}

\section{Patients and tissue samples}

A total of 44 CRC patients who were treated at Shanghai East Hospital from October 2009 until April 2010 were enrolled in the study. Surgical specimens of cancerous tissue, paired adjacent noncancerous tissue and lymph node were obtained from all cases, in accordance with the Ethics Committee of Shanghai East Hospital after obtaining an informed consent. Patients' data on age, tumor sites, multiplicity, histologic grade, invasion depth, presence of lymph node metastasis, necrosis, lymphatic and the number stage stages were collected. The sample included 27 men and 17 women, with a mean age of 67 years (median 67 years; in women 63 years; in men 68 years; range 43-91 years). Histological tumor staging was performed by routine pathology. The detailed clinicopathological characteristics are summarized in Table 2. All tissue samples were incubated in RNA Later (Ambion, Austin, Texas, USA) at $4^{\circ} \mathrm{C}$ for 24 hours and stored at $-80^{\circ} \mathrm{C}$ until RNA extraction.

\section{Measurement of STK31 expression}

\section{Total RNA extraction and reverse transcription}

Total RNA isolated from tumor, adjacent normal tissue and lymph node samples was prepared with Trizol Reagent (Invitrogen, Carlsbad, CA, USA), according to manufacturer's protocol. Reverse transcription was performed with $1 \mu \mathrm{g}$ total RNA and MMLV reverse transcriptase (Promega, Madison, Wisconsin, USA). cDNA was stored at $-80^{\circ} \mathrm{C}$.

\section{Quantitative real-time polymerase chain reaction (QRT-PCR)}

Reactions were performed using Syber green PCR Master Mix (Takara Biotech, Dalian, China) in real-time PCR apparatus (iCycler iQ5, Bio-Rad, Hercules, CA, USA). 
Sense primer 5'-AAATCTGTGAGTCAGCGAGCCT-3' and antisense primer 5'-TGATCCACTTTGGGGATTCC AT-3' were used to amplify STK31 gene; glyceraldehyde3-phosphate dehydrogenase (GAPDH; sense primer 5'-AGAAGGCTGGGGCTCATTTG-3' and antisense primer 5'- AGGGGCCATCCACAGTCTTC - $3^{\prime}$ ) as internal control. Primers were synthesized by Sangon Biotech (Shanghai) Co., Ltd. (China). The QRT-PCR was performed in strict accordance with the kit instructions. Thermal cycling for all reactions was initiated with a denaturation step at $95^{\circ} \mathrm{C}$ for $10 \mathrm{~min}$, followed by 40 cycles at $95^{\circ} \mathrm{C}$ for $5 \mathrm{~s}$ and $60^{\circ} \mathrm{C}$ for $45 \mathrm{~s}$. Each assay was performed as triplicate. Results were quantitated using comparative cycle threshold (CT) method $2^{-\Delta \mathrm{CT}}$ (You et al. 2010). All calculated concentrations of the target gene were divided by endogenous reference (GAPDH) to obtain normalized STK31 expression values.

\section{Immunohistochemistry}

In brief, formalin-fixed, paraffin-embedded tissue sections were dewaxed and the antigens retrieved. The primary antibody against STK31 (abcam, USA) was diluted 1:40. Secondary anti-mouse antibodies were applied on slides for $1 \mathrm{~h}$ at $37^{\circ} \mathrm{C}$, followed by diaminobenzidine and hematoxylin treatment. PBS was used in the same manner as a substitute for the primary antibody and used as negative control.

\section{Statistical analysis}

Statistical calculations were performed using SPSS version 17.0 (SPSS, Chicago, IL). Differences between groups were estimated using Student's $t$ test (IndependentSamples $t$ test or Paired-Samples $t$ test) and One-way ANOVA analysis. A two-sided significance level of 0.05 (confidence level $>95 \%$ ) was used for all statistical tests.

\section{ACKNOWLEDGMENTS AND FUNDING}

This study was sponsored by Academic Leaders Training Program of Pudong Health Bureau of Shanghai (Grant No. PEWd2010-05) and Research Grant for Health Science and Technology of Pudong Health Bureau of Shanghai (Grant No. PKJ2008-38).

\section{CONFLICTS OF INTEREST}

There is no conflicts of interest in connection with this manuscript.

\section{REFERENCES}

1. Kanojia D, Garg M, Gupta S, Gupta A, Suri A. Spermassociated antigen 9 is a novel biomarker for colorectal cancer and is involved in tumor growth and tumorigenicity. Am J Pathol. 2011; 178:1009-20.
2. Compton CC. Colorectal carcinoma: diagnostic, prognostic, and molecular features. Modern Pathol. 2003; 16:376-88.

3. Walsh JM, Terdiman JP. Colorectal cancer screening: scientific review. JAMA. 2003; 289:1288-96.

4. You S, Zhou J, Chen S, Zhou P, Lv J, Han X, Sun Y. PTCH1, a receptor of Hedgehog signaling pathway, is correlated with metastatic potential of colorectal cancer. Ups J Med Sci. 2010; 115:169-75.

5. Wang PJ, McCarrey Jr, Yang F, Page DC. An abundance of X-linked genes expressed in spermatogonia. Nat Genet. 2001; 27:422-6.

6. Wang PJ, Page DC, McCarrey JR. Differential expression of sex-linked and autosomal germ-cell-specific genes during spermatogenesis in the mouse. Hum Mol Genet. 2005; 14:2911-8.

7. Sabeur K, Ball BA, Corbin CJ, Conley A. Characterization of a novel, testis-specific equine serine/threonine kinase. Mol Reprod Dev. 2008; 75:867-73.

8. Ponting CP. Tudor domains in proteins that interact with RNA. Trends Biochem Sci. 1997; 22:51-2.

9. Leach DRF, Connelly JC, Kirkham LA. The SbcCD nuclease of Escherichia coli is a structural maintenance of chromosomes (SMC) family protein that cleaves hairpin DNA. P Natl Acad Sci USA. 1998; 95:7969-74.

10. Spiridonov NA, Wong L, Zerfas PM, Starost MF, Pack SD, Paweletz CP, Johnson GR. Identification and characterization of SSTK, a serine/threonine protein kinase essential for male fertility. Mol Cell Biol. 2005; 25:4250-61.

11. Means AR, Wu JY, Ribar TJ, Cummings DE, Burton KA, McKnight GS. Spermiogenesis and exchange of basic nuclear proteins are impaired in male germ cells lacking Camk4. Nat Genet. 2000; 25:448-52.

12. Mori M, Yokoe $\mathrm{T}$, Tanaka $\mathrm{F}$, Mimori $\mathrm{K}$, Inoue $\mathrm{H}$, Ohmachi T, Kusunoki M, Mori M. Efficient identification of a novel cancer/testis antigen for immunotherapy using three-step microarray analysis. Cancer Res. 2008; $68: 1074-82$.

13. Brown GJ, Saunders BP. Advances in colonic imaging: technical improvements in colonoscopy. Eur J Gastroenterol Hepatol. 2005; 17:785-92.

14. Hughes SJ, Hriesik C, Ramanathan RK. Update for surgeons: Recent and noteworthy changes in therapeutic regimens for cancer of the colon and rectum. J Am Coll Surgeons. 2007; 205:468-78.

15. Hurwitz H, Fehrenbacher L, Novotny W, Cartwright T, Hainsworth J, Heim W, Berlin J, Baron A, Griffing S, Holmgren E, Ferrara N, Fyfe G, Rogers B, et al. Bevacizumab plus irinotecan, fluorouracil, and leucovorin for metastatic colorectal cancer. N Engl J Med. 2004; 350:2335-42.

16. Benson $\mathrm{AB} 3 \mathrm{rd}$. Epidemiology, disease progression, and economic burden of colorectal cancer. J Manag Care Pharm. 2007; 13:S5-18. 
17. Stein U, Schlag PM. Clinical, biological, and molecular aspects of metastasis in colorectal cancer. Recent Results Cancer Res. 2007; 176:61-80.

18. Christofori G. New signals from the invasive front. Nature. 2006; 441:444-50.

19. Nordlinger B, Penna C. Colorectal metastasis (liver and lung). Surg Clin N Am. 2002; 82:1075-90.

20. Stein U, Burock S, Herrmann P, Wendler I, Niederstrasser M, Wernecke KD, Schlag PM. Diagnostic and prognostic value of metastasis inducer S100A4 transcripts in plasma of colon, rectal, and gastric cancer patients. J Mol Diagn. 2011; 13:189-98.

21. Beahrs OH. Staging of cancer of the colon and rectum. Cancer. 1992; 70:1393-6.
22. Carpelan-Holmstrom M, Louhimo J, Stenman UH, Alfthan H, Haglund C. CEA, CA 19-9 and CA 72-4 improve the diagnostic accuracy in gastrointestinal cancers. Anticancer Res. 2002; 22:2311-6.

23. Fok KL, Chung CM, Yi SQ, Jiang X, Sun X, Chen H, Chen YC, Kung HF, Tao Q, Diao R, Chan H, Zhang XH, Chung YW, et al. STK31 maintains the undifferentiated state of colon cancer cells. Carcinogenesis. 2012; 33:2044-53.

24. Kuo PL, Huang YL, Hsieh CC, Lee JC, Lin BW, Hung LY. STK31 is a cell-cycle regulated protein that contributes to the tumorigenicity of epithelial cancer cells. PLoS One. 2014; 9:e93303. doi:10.1371/journal.pone.0093303.

25. Campos SM, Dizon DS. Antimitotic inhibitors. Hematol Oncol Clin North Am. 2012; 26:607-28. 